\title{
Electron (positron) beam polarization by Compton scattering on circularly polarized laser photons
}

\author{
G. L. Kotkin, ${ }^{1}$ V. G. Serbo, ${ }^{1}$ and V. I. Telnov ${ }^{2}$ \\ ${ }^{1}$ Novosibirsk State University, 630090, Novosibirsk, Russia \\ ${ }^{2}$ Budker Institute of Nuclear Physics, 630090, Novosibirsk, Russia
}

(Received 14 June 2002; published 14 January 2003)

\begin{abstract}
In a number of papers an attractive method of laser polarization of electrons (positrons) at storage rings or linear colliders has been proposed. We show that these suggestions are incorrect and based on errors in the simulation of multiple Compton scattering and in the calculation of the Compton spin-flip cross sections. We argue that the equilibrium polarization in this method is zero.
\end{abstract}

DOI: 10.1103/PhysRevSTAB.6.011001

PACS numbers: 41.75.Ht, 29.17.+w, 29.27.Hj, 13.60.Fz

\section{INTRODUCTION}

Experiments at SLC have shown great potential of polarized $e^{ \pm}$beams for investigation of new physical phenomena. In all projects of future $e^{+} e^{-}, e^{-} e^{-}, \gamma \gamma$, and $\gamma e$ linear colliders [1], electron and positron beams with a high degree of polarization are foreseen, though this is not an easy task. That is why any new methods for obtaining polarized $e^{ \pm}$beams are very welcome.

There are two well-known and recognized methods for production of polarized beams for linear colliders. In the first method, electron beams with a polarization of $80 \%$ (maybe even higher) are obtained using photoguns [2]. Another method of polarization, suitable for both electron and positron beams, is based on a two-step scheme [3]. At the first step, the unpolarized electron beam passes through a helical undulator (or collides with circularly polarized laser light) and produces photons with maximum energy of about $30-50 \mathrm{MeV}$. These photons have a high degree of circular polarization in the high energy part of the spectrum. Then these photons pass through a thin tungsten target and produce $e^{+} e^{-}$ pairs. At the maximum energies, these particles have a high degree of longitudinal polarization. The expected polarization of electron and positron beams in this method is $45 \%-60 \%[4,5]$.

Additionally, in a number of papers a new attractive $\operatorname{method}^{1}$ for production of polarized electron beams, based on the process of multiple Compton scattering of ultrarelativistic electrons on the circularly polarized laser photons, was discussed. Here one should distinguish between two possibilities: polarization of the beam at the cost of a loss in intensity and polarization without loss of intensity. The latter case is the subject of the present paper.

It is well known that in Compton scattering the electrons of different helicities are knocked out of the beam

\footnotetext{
${ }^{1}$ This method is applicable both for electrons and positrons; for brevity we will omit "positrons" hereafter.
}

differently. As a consequence, after removal of the scattered electrons, the electron beam can get a considerable polarization at the expense of a considerable loss of its intensity. A detailed consideration of this method was given in [6]. Though the basic idea of this method is correct, it was not used in practice because the losses in intensity during the polarization process are too large.

In this paper we critically consider another proposal of beam polarization which is based on multiple Compton scattering of electrons on laser photons without loss of intensity. It implies that during a single Compton scattering the energy loss of an electron is small and the electron scattering angle is small as well; therefore, the scattered electrons remain in the beam. It means that the electron energy is of the order of $1 \mathrm{GeV}$ for laser light with a photon energy of about $1 \mathrm{eV}$. Such proposals were given in papers [7-10]. They were cited in a number of papers (see Refs. [11], for example) and attracted attention at the Snowmass 2001 conference. It was suggested to implement this method either on a storage ring (where the electron beam collides with laser beams many times at a single point) or at a linear collider (where the electron beam would collide with laser beams at several points) with reacceleration between them.

The theoretical consideration of the process of multiple Compton scattering in papers [7-10] is based on two different approaches. The first approach exploits the fact that the scattered electrons are polarized even if the initial electron beam is unpolarized. Since the scattered electrons do not leave the beam, multiple collisions with laser photons would appear to lead to a growth of the mean electron beam polarization. The quantitative consideration of this idea in papers [8,9] resulted in the following.

Conclusion 1 [8,9]: A longitudinal polarization of electrons (positrons) up to $100 \%$ can be achieved in a relatively short time.-In Sec. III we explain the origin of the mistake that lead to conclusion 1. Briefly speaking, in simulation of multiple Compton scattering one should not only consider the multiple Compton scattering of the 
same electron but also take into account the fact that the polarization of unscattered electrons changes in the laser wave as well. The correct simulation procedure for multiple Compton scattering (Sec. II) leads to zero polarization of the final electron beam.

In the second approach, only the equilibrium polarization of the electron beam after multiple passes through the laser beam was considered. Let $w_{+-}$and $w_{-+}$be the probabilities for Compton scattering with a given electron spin flip. It is not difficult to show (see Sec. IIB) that the electron beam gets the maximal equilibrium polarization $\zeta_{z}^{(f)}=\left(w_{-+}-w_{+-}\right) /\left(w_{-+}+w_{+-}\right)$. The corresponding probabilities have been calculated in papers $[7,10]$ with the following.

Conclusion 2 [7,10]: The longitudinal polarization of electrons (positrons) as large as $62.5 \%$ can be achieved.-In Sec. IV we show that conclusion 2 is due to an error in the calculation of the Compton spin-flip cross sections. The error is connected to the incorrect transition between the collider frame (CF) and the rest frame of the initial electron (RFIE). The correct result corresponds to $w_{+-}=w_{-+}$, therefore, the discussed process of laser polarization is impossible.

Below, in Sec. II, we present a set of formulas for Compton scattering that takes into account the particle polarization from Ref. [12], as well as a short description of the simulation procedure for the multiple Compton scattering from Ref. [13], which are useful for quantitative consideration of this method. Here we show that scattering of laser photons does not lead to polarization of electron beams; in fact it leads only to depolarization. In Secs. III and IV we explicitly show the origin of mistakes in the previous papers on this subject.

\section{POLARIZATION OF FINAL ELECTRONS}

\section{A. Polarization of final electrons in single Compton scattering}

We consider the basic Compton scattering

$$
e(p)+\gamma(k) \rightarrow e\left(p^{\prime}\right)+\gamma\left(k^{\prime}\right)
$$

in the CF, in which an electron with energy $E \sim 1 \mathrm{GeV}$ collides a head-on with a laser photon of energy $\omega \sim 1 \mathrm{eV}$.

Let us introduce some notation related to the Compton scattering (1) in the CF. We choose the quantization axis $(z$ axis) along the initial electron momentum $\mathbf{p}$ (i.e., antiparallel to the laser photon momentum k). Let $P_{c}=$ +1 be the mean helicity of the circularly polarized laser photons, and $\zeta=\left(0,0, \zeta_{z}\right)$ and $\zeta^{\prime}=\left(0,0, \zeta_{z}^{\prime}\right)$ be the polarization vectors of the electron in the initial and final states. It is convenient to describe the Compton scattering by the invariants $(c=1, \hbar=1)$

$$
x=\frac{2 p k}{m_{e}^{2}}, \quad y=\frac{2 p k^{\prime}}{p k}=\frac{2 p^{\prime} k}{p k} .
$$

In $\mathrm{CF}$, we have

$$
x=\frac{4 E \omega}{m_{e}^{2}}, \quad y=\frac{\omega^{\prime}}{E} .
$$

The maximum energy of scattered photons is

$$
\max \left\{\omega^{\prime}\right\}=y_{m} E, \quad y_{m}=\frac{x}{x+1} .
$$

For $E \sim 1 \mathrm{GeV}$ and $\omega \sim 1 \mathrm{eV}$, the value of $x \sim 0.015$, therefore hereafter we assume

$$
x \ll 1, \quad E-E^{\prime}=\omega^{\prime} \ll E .
$$

In RFIE, the energy of the laser photon $x m_{e} / 2$ is small in comparison with the electron mass: $x m_{e} / 2 \ll m_{e}$; and therefore the transverse momenta are small as well:

$$
\left|\mathbf{p}_{\perp}^{\prime}\right|=\left|\mathbf{k}_{\perp}^{\prime}\right|<x m_{e} / 2 \ll m_{e} .
$$

As a result, the electron scattering angle in the CF is very small

$$
\theta_{e} \approx \frac{\left|\mathbf{p}_{\perp}^{\prime}\right|}{p_{z}^{\prime}}<\frac{x m_{e}}{2 E} \approx \frac{2 \omega}{m_{e}} \sim 4 \times 10^{-6}
$$

The Compton cross section in the collider frame for the above conditions has been given in [12]:

$$
\begin{gathered}
\frac{d \sigma}{d y}=\frac{\pi r_{e}^{2}}{x}\left[\left(1+\zeta_{z} \zeta_{z}^{\prime}\right) F_{1}+\left(\zeta_{z}+\zeta_{z}^{\prime}\right) P_{c} F_{2}+\zeta_{z} \zeta_{z}^{\prime} F_{3}\right], \\
F_{1}=\frac{1}{1-y}+c^{2}-y, \quad F_{2}=\frac{y(2-y) c}{1-y}, \\
F_{3}=-\frac{y^{2} s^{2}}{1-y},
\end{gathered}
$$

where $r_{e}$ is the classical electron radius and

$s=2 \sqrt{r(1-r)}, \quad c=1-2 r, \quad r=\frac{y}{(1-y) x}$

(in RFIE, the invariant $s=\sin \vartheta$ and the invariant $c=$ $\cos \vartheta$, where $\vartheta$ is the photon scattering angle). ${ }^{2}$

\section{B. Balance equations}

If we do not take into account the transverse polarization of electrons, we can describe the electron beam as a mixture of $N_{+}$electrons with $\zeta_{z}=+1$ and $N_{-}$electrons with $\zeta_{z}=-1$. In this case the mean value of the longitudinal electron polarization is

\footnotetext{
${ }^{2}$ Strictly speaking, Eq. (8) given in [12] is valid for the case when $\zeta_{z}^{\prime}$ is the projection of the vector $\zeta^{\prime}$ on the momentum of the final electron $\mathbf{p}^{\prime}$. However, the projections of $\boldsymbol{\zeta}^{\prime}$ on $\mathbf{p}^{\prime}$ and on the $z$ axis coincide in our approximation due to the very small value of the electron scattering angle in the CF (7).
} 


$$
\zeta_{z}=\frac{N_{+}-N_{-}}{N_{+}+N_{-}}
$$

When the electron beam travels the path $d z$ in the laser beam with the laser photon density $n_{L}(z)$, the change of numbers $N_{ \pm}(z)$ is given by the balance equations derived by the following simple consideration. A reduction of the number of electrons with $\zeta_{z}=+1$ in the beam is determined by the quantity $N_{+}(z)\left(d w_{++}+d w_{+_{-}}\right)$, where

$$
d w_{\zeta_{z} \zeta_{z}^{\prime}}=2 \sigma\left(\zeta_{z}, \zeta_{z}^{\prime}\right) n_{L}(z) d z
$$

is the probability that an electron with a certain $\zeta_{z}$ is scattered on the path $d z$ with the transition to a certain $\zeta_{z}^{\prime}$ (here we assume also a certain $P_{c}=+1$ ). The coefficient 2 is due to the fact that the electron and the laser photon travel towards each other with the speed of light. On the other hand, the sum $N_{+}(z) d w_{++}+N_{-}(z) d w_{-+}$represents the number of scattered electrons with $\zeta_{z}^{\prime}=+1$.

As a result, the total change of the number of electrons with $\zeta_{z}=+1$ is equal to

$$
\begin{aligned}
d N_{+}(z)= & -N_{+}(z)\left(d w_{++}+d w_{+-}\right)+N_{+}(z) d w_{++} \\
& +N_{-}(z) d w_{-+} \\
= & -N_{+}(z) d w_{+-}+N_{-}(z) d w_{-+},
\end{aligned}
$$

and, similarly,

$$
d N_{-}(z)=-N_{-}(z) d w_{-+}+N_{+}(z) d w_{+-} .
$$

Since in the considered method the scattered electrons remain in the beam, the sum $N_{+}+N_{-}=N_{e}$ does not change:

$$
d N_{+}(z)+d N_{-}(z)=0,
$$

while the mean longitudinal polarization (10), generally speaking, changes as

$$
\begin{aligned}
N_{e} d \zeta_{z} & =d N_{+}(z)-d N_{-}(z) \\
& =-2 N_{+}(z) d w_{+-}+2 N_{-}(z) d w_{-+} .
\end{aligned}
$$

Balance equations (12)-(15) are simplified in two particular cases. First, if the initial electron beam is unpolarized, $N_{+}(z)=N_{-}(z)=N_{e} / 2$, it gets (after traveling the path $d z$ ) the polarization

$$
d \zeta_{z}=d w_{-+}-d w_{+-} .
$$

Second, let us consider the equilibrium polarization of the electron beam (which is achieved after multiple passing of the electron beam through the laser beam). In this case, $d N_{+}=d N_{-}=0$ or

$$
N_{+} d w_{+-}=N_{-} d w_{-+} .
$$

From this equation one obtains the equilibrium polarization degree of the electron beam

$$
\zeta_{z}^{(f)}=\frac{w_{-+}-w_{+-}}{w_{-+}+w_{+-}}
$$

where $w_{ \pm \mp}=\int d w_{ \pm \mp}$ is the probability for the whole path in the laser beam. The quantity $w_{+-}-w_{-+}$is proportional to the difference of the Compton spin-flip cross sections (with a certain $P_{c}=+1$ and with the summation over spin states of the final photon):

$$
\Delta \sigma=\sigma\left(\zeta_{z}=+1, \zeta_{z}^{\prime}=-1\right)-\sigma\left(\zeta_{z}=-1, \zeta_{z}^{\prime}=+1\right)
$$

Therefore, in both of these cases the crucial question is whether $\Delta \sigma$ is equal to zero or not equal to zero.

In papers $[7,10]$ it was claimed that the difference

$$
\Delta \sigma \neq 0,
$$

the process of laser polarization of particles is possible and $\zeta_{z}^{(f)}=5 / 8$. In contrast, using formulas (8) we immediately obtain

$$
\Delta \sigma=0,
$$

and, therefore, the polarization is zero. ${ }^{3}$ The origin of the incorrect result (20) is explained in Sec. IV.

Taking into account that $d w_{+-}=d w_{-+}$, we can rewrite Eq. (15) in the form

$$
\frac{d \zeta_{z}}{\zeta_{z}}=-4 \sigma\left(\zeta_{z}=+1, \zeta_{z}^{\prime}=-1\right) n_{L}(z) d z
$$

from which it follows that the polarization $\left|\zeta_{z}\right|$ is reduced after traveling the path $d z$.

Note that the result (21) is due to the specific structure of Eq. (8): the coefficient in front of $\zeta_{z} P_{c}$ in this equation precisely coincides with the coefficient in front of $\zeta_{z}^{l} P_{c}$.

\section{A scheme for simulation of multiple Compton scattering}

In some problems such as conversion of electrons to photons at photon colliders, laser cooling, etc., it is necessary to calculate beam parameters after multiple acts of Compton scattering. Let an electron beam traverse a region where laser light is focused. It is clear that the energies of these electrons as well as their polarizations vary due to Compton scattering.

However, when the electron passes through the laser beam, the polarization varies also for those electrons which conserve their energies and directions of motion (unscattered electrons). This effect is due to the interference of the incoming electron wave and the electron wave scattered at zero angle. The change in the electron polarization depends not only on the Compton cross section but on the real part of the forward Compton amplitude as well. Such an effect was considered in Ref. [13].

Both of these effects should be taken into account in simulation of multiple Compton scattering. It can be taken into account in the following way. The electron

\footnotetext{
${ }^{3}$ The similar remark about the equal spin-flip probabilities in the CF was given (without details) in Ref. [14].
} 
state is defined by the current values of its energy $E$, the direction of its momentum (along the $z$ axis), and its mean polarization vector $\zeta$. The probability to scatter on the path $d z$ is equal to

$$
d w=2 \sigma\left(E, \zeta_{z}\right) n_{L}(z) d z
$$

where $\sigma\left(E, \zeta_{z}\right)$ is the total cross section of the Compton scattering process. Then, as usual, one can simulate whether the scattering takes place on this path $d z$ or not.

If the scattering does take place, then, using known formulas for the Compton cross section in the CF (see Ref. [12]), one can calculate a new value of the electron polarization vector $\boldsymbol{\zeta}^{(f)}$ and other parameters.

If the scattering does not occur, one still has to change the electron polarization vector. ${ }^{4}$ The variation of electron polarization in the laser wave for a general case was considered in [13]. Following that paper, the change of the electron polarization vector of the unscattered electron is

$$
\begin{gathered}
d \zeta_{x}=\left(R \zeta_{y}+I \zeta_{z} \zeta_{x}\right) P_{c} 2 \pi r_{e}^{2} n_{L} d z, \\
d \zeta_{y}=\left(-R \zeta_{x}+I \zeta_{z} \zeta_{y}\right) P_{c} 2 \pi r_{e}^{2} n_{L} d z, \\
d \zeta_{z}=-I\left(1-\zeta_{z}^{2}\right) P_{c} 2 \pi r_{e}^{2} n_{L} d z,
\end{gathered}
$$

where the functions $I=I(x)$ and $R=R(x)$ are equal to

$$
\begin{gathered}
I=\frac{2}{x} \int_{0}^{x /(1+x)} \frac{y(2-y) c}{1-y} d y \\
=\frac{2}{x}\left[\left(1+\frac{2}{x}\right) \ln (x+1)-\frac{5}{2}+\frac{1}{x+1}-\frac{1}{2(x+1)^{2}}\right] \\
R(x)=\frac{2}{\pi x}\left[\left(1-\frac{2}{x}\right) F(x-1)-\left(1+\frac{2}{x}\right) F(-x-1)\right. \\
\left.\quad-\frac{2 x^{3} \ln x}{\left(x^{2}-1\right)^{2}}+\frac{x}{x^{2}-1}-\frac{2 \pi^{2}}{3 x}\right]
\end{gathered}
$$

with

$$
F(x)=\int_{0}^{x} \frac{\ln |1+t|}{t} d t
$$

being the Spence function.

\footnotetext{
${ }^{4}$ The necessity of this step can also be seen from the following consideration. The value of the Compton cross section depends on polarizations of electron and laser beams. If the electron beam was initially unpolarized, then, after the Compton scattering of one electron, the rest (unscattered) part of the beam gets some polarization (see Sec. IIB). That is just because electrons with different polarizations have different scattering probabilities. In other words, the laser beam "selects" preferably electrons with a certain polarization. In particular, Eq. (26) for the longitudinal polarization can be obtained from the balance equations discussed above.
}

Now we can calculate the total change of the electron beam polarization on the distance $d z$ in the laser target by the means of simulation.

If the initial electron is unpolarized $\left(\zeta_{z}=0\right)$ and the laser photon is circularly polarized $\left(P_{c}=+1\right)$, then from Eq. (8) we have

$$
\sigma=\frac{1}{2}\left[\sigma_{\mathrm{unpol}}+\pi r_{e}^{2} I(x) \zeta_{z}^{\prime}\right]
$$

where $\sigma_{\text {unpol }}$ is the Compton cross section for unpolarized beams. Therefore, the scattered electron becomes polarized after the first scattering, and its mean degree of polarization is

$$
\zeta_{z}^{(f)}=\frac{\pi r_{e}^{2} I(x)}{\sigma_{\text {unpol }}} .
$$

The total number of scattered electrons on the path $d z$ in the laser target is

$$
d N_{e}=2 \sigma_{\text {unpol }} N_{e} n_{L} d z .
$$

This means that the sum of polarizations of the scattered electrons along the $z$ axis:

$$
\zeta_{z}^{(f)} d N_{e}=2 \pi r_{e}^{2} I(x) N_{e} n_{L} d z
$$

On the other hand, the unscattered electrons become polarized in accordance with Eq. (26), and their sum of polarizations along the $z$ axis,

$$
d \zeta_{z} N_{e}=-2 \pi r_{e}^{2} I(x) N_{e} n_{L} d z
$$

i.e., it completely compensates the above polarization of the scattered electrons. So, the electron beam remains unpolarized. This coincides with our result obtained in Sec. IIB where only the equilibrium final state was considered.

At the end of this subsection we note the following. The components of the vector $\zeta$ define the parameters of the polarization density matrix of an electron, among them, $\zeta_{z}$ is related to the diagonal matrix elements while $\zeta_{x}$ and $\zeta_{y}$ determine the off-diagonal matrix elements. If one is interested in the longitudinal electron polarization only (as in the present paper), it is sufficient in simulation to use Eq. (26) connected with the "occupancy" numbers $N_{ \pm}$. In the general case one should use the whole set of Eqs. (24)-(26).

\section{REMARK ON CONCLUSION 1}

Now we are ready to show the origin of the error in conclusion 1. Let us describe the procedure of the naive simulation of the multiple Compton scattering. We consider the case when in the CF the polarization vectors of the initial and final electrons have $z$ components only and the parameter $x$ is small. The corresponding Compton cross section with an accuracy up to the terms of the order of $x$ can be easily obtained from (8): 


$$
\sigma=\frac{4}{3} \pi r_{e}^{2}\left[(1-x)\left(1+\zeta_{z} \zeta_{z}^{\prime}\right)-\frac{x}{4} P_{c}\left(\zeta_{z}+\zeta_{z}^{\prime}\right)\right]
$$

If the initial electron is unpolarized $\left(\zeta_{z}=0\right)$ and the laser photon is circularly polarized $\left(P_{c}=+1\right)$, then

$$
\sigma=\frac{4}{3} \pi r_{e}^{2}\left[1-x-\frac{x}{4} \zeta_{z}^{\prime}\right]
$$

i.e., the cross section is somewhat larger for $\zeta_{z}^{\prime}=-1$ than for $\zeta_{z}^{\prime}=+1$. Therefore, the scattered electron becomes polarized after the first scattering and its mean degree of polarization is

$$
\zeta_{z}^{(f)}=-\frac{x}{4}
$$

Repeating this procedure, one can find that after $N$ collisions the electron polarization is

$$
\zeta_{z}^{(f)}=-\frac{N}{(4 / x)+N},
$$

which can reach $100 \%$ for $N \gg 4 / x$. This fact is the basis for conclusion 1.

This "polarization" is not connected with the electron spin flip, it is due to some differences in the cross sections: the polarized laser beam selects electrons with a certain (in our case, negative) polarization. But such a naive simulation of the multiple Compton scattering is incorrect because it does not take into account the fact that unscattered electrons become polarized in the opposite direction. The correct procedure for this simulation is described in the previous section and leads to zero polarization.

\section{REMARK ON CONCLUSION 2}

In Sec. IIA we have shown that the equilibrium polarization of electrons in the discussed method is zero. Below, we show the origin of the mistake that led to conclusion 2. We remind that our result (21) has been obtained in the CF. To the contrary, the authors of conclusion 2 had obtained their result (20) in the RFIE. Below we demonstrate how to obtain our result in RFIE and show that the error in conclusion 2 is connected with an inaccurate transition from the CF to RFIE.

In our consideration, we use the electron polarization vectors $^{5} \zeta$ and $\boldsymbol{\zeta}^{\prime}$, which in the CF have the forms

$$
\zeta=(0,0, \pm 1), \quad \zeta^{\prime}=(0,0, \mp 1)
$$

It is not difficult to show that in RFIE the vector $\zeta$ has the same form, but the vector $\zeta^{\prime}$ has another form:

\footnotetext{
${ }^{5}$ They determine the electron-spin 4-vectors $a$ and $a^{\prime}$ as $a=$ $\left(\boldsymbol{\zeta} \mathbf{p} / m_{e}\right), \boldsymbol{\zeta}+\mathbf{p}\left[\boldsymbol{\zeta} \mathbf{p} / m_{e}\left(E+m_{e}\right)\right], \quad a^{\prime}=\left(\boldsymbol{\zeta}^{\prime} \mathbf{p}^{\prime}\right) / m_{e}, \boldsymbol{\zeta}^{\prime}+$ $\mathbf{p}^{\prime}\left[\zeta^{\prime} \mathbf{p}^{\prime} / m_{e}\left(E^{\prime}+m_{e}\right)\right]$.
}

$$
\boldsymbol{\zeta}_{\perp}^{\prime}=\mp \frac{\mathbf{p}_{\perp}^{\prime}}{m_{e}}= \pm \frac{\mathbf{k}_{\perp}^{\prime}}{m_{e}}, \quad \zeta_{z}^{\prime}=\mp\left(1-\frac{\left(\mathbf{p}_{\perp}^{\prime}\right)^{2}}{2 m_{e}^{2}}\right) \approx \mp 1,
$$

since transition from the CF to RFIE corresponds to a boost along the vector $\mathbf{p}$, but not along the vector $\mathbf{p}^{\prime}$. To prove (40), it is sufficient to consider the transverse component of the electron-spin 4-vector $a^{\prime}$ in the $\mathrm{CF}$ and in the RFIE. In the CF, one has

$$
\mathbf{a}_{\perp}^{\prime}=\mp \mathbf{p}_{\perp}^{\prime} \frac{p_{z}^{\prime}}{m_{e}\left(E^{\prime}+m_{e}\right)} \approx \mp \frac{\mathbf{p}_{\perp}^{\prime}}{m_{e}},
$$

since in the CF we have $p_{z}^{\prime} /\left(E^{\prime}+m_{e}\right) \approx 1$. In RFIE the same polarization vector is

$$
\mathbf{a}_{\perp}^{\prime}=\zeta_{\perp}^{\prime}+\mathbf{p}_{\perp}^{\prime} \frac{\zeta^{\prime} \mathbf{p}^{\prime}}{m_{e}\left(E^{\prime}+m_{e}\right)} \approx \zeta_{\perp}^{\prime},
$$

since in RFIE we have $\left|\mathbf{p}^{\prime}\right| / m_{e} \ll 1$.

The needed Compton cross section in RFIE can be found in the textbook [15] [see Eqs. $(87,22)$ and $(87,23)]$ :

$$
\frac{d \sigma}{d \Omega}=\frac{r_{e}^{2}}{4}\left(\frac{\omega^{\prime}}{\omega}\right)^{2}\left[F_{0}+\mathbf{f} \zeta P_{c}+\mathbf{g} \zeta^{\prime} P_{c}+G_{i k} \zeta_{i} \zeta_{k}^{\prime}\right]
$$

From this we get the following result:

$$
\frac{d \Delta \sigma}{d \Omega}=\frac{r_{e}^{2}}{2}\left(\frac{\omega^{\prime}}{\omega}\right)^{2}\left[f_{z}-g_{z}+\mathbf{g}_{\perp} \frac{\mathbf{k}_{\perp}^{\prime}}{m_{e}}\right] .
$$

Before proceeding, we note that the momenta of the initial and final photons in RFIE are

$$
\mathbf{k}=(0,0,-\omega), \quad \mathbf{k}^{\prime}=\left(\mathbf{k}_{\perp}^{\prime},-\omega^{\prime} \cos \vartheta\right),
$$

where $\vartheta$ is the photon scattering angle (the direction of the $z$ axis is along the vector $(-\mathbf{k}))$. Using Eq. $(87,23)$ from [15] and taking into account the terms of the second order in $\omega / m_{e}$ we obtain

$$
\begin{gathered}
f_{z}-g_{z}=\frac{\omega-\omega^{\prime} \cos \vartheta}{m_{e}} \frac{\omega+\omega^{\prime}}{2 m+\omega-\omega^{\prime}} \sin ^{2} \vartheta \\
\approx\left(\frac{\omega}{m_{e}}\right)^{2}(1-\cos \vartheta) \sin ^{2} \vartheta \\
\mathbf{g}_{\perp} \frac{\mathbf{k}_{\perp}^{\prime}}{m_{e}}=-\frac{\left(\mathbf{k}_{\perp}^{\prime}\right)^{2}}{m_{e}^{2}}(1-\cos \vartheta) \sin ^{2} \vartheta \\
\\
\approx-\left(\frac{\omega}{m_{e}}\right)^{2}(1-\cos \vartheta) \sin ^{2} \vartheta .
\end{gathered}
$$

As a result, we get

$$
\frac{d \Delta \sigma}{d \Omega}=0
$$

which is in agreement with the conclusion (21) in the CF.

The wrong conclusion (20) was obtained because the same form (39) was used for the vector $\zeta^{\prime}$ both in the CF 
and in the RFIE. It is equivalent to omitting the last term in the square bracket in Eq. (44).

Thus, the calculations, performed in $\mathrm{CF}$ as well as in RFIE, give us the same result (21). We, therefore, conclude that the claim (20) is based on an inaccurate transition from the CF to the RFIE.

\section{SUMMARY}

We have shown that the multiple Compton scattering of electrons on circularly polarized laser photons at usual storage rings or linear accelerators does not lead to polarization of electron beams. Statements by some authors about obtainability of high degrees of polarization are explained by mistakes in their calculation procedures. We had discussions with Bessonov and Potylitsyn, and they agreed with our criticism.

In this paper we have considered the linear Compton scattering (the scattering of an electron on a single laser photon). It is technically possible to realize conditions which correspond to the nonlinear Compton scattering (the scattering of an electron on several laser photons). The effective cross section for the nonlinear Compton scattering from Ref. [16] has the same specific structure as Eq. (8) but with much more complicated functions $F_{1,2,3}$. From this, one can easily obtain the result (21), which means that the equilibrium polarization of electrons is zero in the case of the nonlinear Compton scattering as well.

One additional remark. There is no polarization of the electron beam as a whole in the considered scheme, however, it does not close the possibility to use lasers for polarization of electron beams in other schemes. For example, it has been shown in Ref. [14] that using specially arranged spin-orbit coupling in damping rings (by adding a solenoid), a polarization of about $60 \%$ may be reached. This method is based on the difference in the Compton cross sections for electrons with different values of their helicities, on the fact that scattered electrons have lower energy compared to unscattered electrons, and on dependence of the spin precession angle on the electron energy. This method is not simple and is too slow for preparation of beams for linear colliders.

\section{ACKNOWLEDGMENTS}

We are very grateful to E. Bessonov, R. Brinkmann, V. Katkov, A. P. Potylitsyn, E. L. Saldin, A. N. Skrinsky, and V. Strakhovenko for useful discussions. This work is supported in part by INTAS (No. 00-00679), RFBR (No. 00-02-17592 and No. 00-15-96691), and by a St. Petersburg grant (No. E00-3.3-146).

[1] NLC, American Linear Collider Working Group, T. Abe et al., SLAC Report No. SLAC-R-570, 2001; TESLA Technical Design Report No. DESY 2001-011, ECFA 2001-209, 2001, edited by F. Richard et al.; JLC, ACFA Linear Collider Working Group, K. Abe et al., KEK Report No. 2001-11, hep-ph/0109166.

[2] J. E. Clendenin et al., in Polarized Gas Targets and Polarized Beams, edited by R. J. Holt and M. A. Miller, AIP Conf. Proc. No. 421 (AIP, New York, 1998), p. 250.

[3] V.E. Balakin and A. A. Mikhailichenko, Budker INP Report No. 79-85, Novosibirsk, 1979.

[4] TESLA Technical Design Report, see [1], part 2.

[5] T. Hirose et al., Nucl. Instrum. Methods Phys. Res., Sect. A 455, 15 (2000); T. Omori, in Proceedings of the LCO2, SLAC, Stanford, 2002 SLAC-WP-21, 2002 (on CD), http://www-conf.slac.stanford.edu/lc02.

[6] Ya. S. Derbenev, A. M. Kondratenko, and E. L. Saldin, Nucl. Instrum. Methods 165, 15 (1979).

[7] Yu. Bashmakov, E. Bessonov, and Ya. Vazdik, Sov. Tech. Phys. Lett. 1, 239 (1975) [Pizma ZhTP 1, 520 (1975)].

[8] R. Rossmanith and R. Schmidt, DESY Internal Report No. M-80/02, 1980.

[9] A. P. Potylitsyn, physics/0001004.

[10] A. P. Potylitsin, in Proceedings of the ICFA Beam Dynamics Workshop on Laser-Beam Interaction, 2001, Stony Brook.

[11] J. E. Clendenin, SLAC Report No. SLAC-PUB8465, 2000, physics/0008018; R. Assmann and F. Zimmerman, CERN Report No. SL-2001-064, 2001; hep-ex/0106058.

[12] G. L. Kotkin, S. I. Polityko, and V. G. Serbo, Nucl. Instrum. Methods Phys. Res., Sect. A 405, 30 (1998).

[13] G. L. Kotkin, H. Perlt, and V. G. Serbo, Nucl. Instrum. Methods Phys. Res., Sect. A 404, 430 (1998).

[14] Ya. S. Derbenev, A. M. Kondratenko, and E. L. Saldin, Nucl. Instrum. Methods 165, 201 (1979).

[15] V. B. Berestetskii, E. M. Lifshitz, and L. P. Pitaevskii, Quantum Electrodynamics (Pergamon Press, New York, 1994), 2nd English edition.

[16] M. Galynskii, E. Kuraev, M. Levchuk, and V. Telnov, Nucl. Instrum. Methods Phys. Res., Sect. A 472, 267 (2001). 